\title{
Efficient and Expressive Knowledge Base Completion Using Subgraph Feature Extraction
}

\author{
Matt Gardner and Tom Mitchell \\ Carnegie Mellon University \\ mglecs.cmu.edu, tom.mitchellecmu.edu
}

\begin{abstract}
We explore some of the practicalities of using random walk inference methods, such as the Path Ranking Algorithm (PRA), for the task of knowledge base completion. We show that the random walk probabilities computed (at great expense) by PRA provide no discernible benefit to performance on this task, so they can safely be dropped. This allows us to define a simpler algorithm for generating feature matrices from graphs, which we call subgraph feature extraction (SFE). In addition to being conceptually simpler than PRA, SFE is much more efficient, reducing computation by an order of magnitude, and more expressive, allowing for much richer features than paths between two nodes in a graph. We show experimentally that this technique gives substantially better performance than PRA and its variants, improving mean average precision from .432 to .528 on a knowledge base completion task using the NELL KB.
\end{abstract}

\section{Introduction}

Knowledge bases (KBs), such as Freebase (Bollacker et al., 2008), NELL (Mitchell et al., 2015), and DBPedia (Mendes et al., 2012) contain large collections of facts about things, people, and places in the world. These knowledge bases are useful for various tasks, including training relation extractors and semantic parsers (Hoffmann et al., 2011; Krishnamurthy and Mitchell, 2012), and question answering (Berant et al., 2013). While these knowledge bases may be very large, they are still quite incomplete, missing large percentages of facts about common or popular entities (West et al., 2014; Choi et al., 2015). The task of knowledge base completion-filling in missing facts by examining the facts already in the $\mathrm{KB}$, or by looking in a corpus - is one attempt to mitigate the problems of this knowledge sparsity.

In this work we examine one method for performing knowledge base completion that is currently in use: the Path Ranking Algorithm (PRA) (Lao et al., 2011; Dong et al., 2014). PRA is a method for performing link prediction in a graph with labeled edges by computing feature matrices over node pairs in the graph. The method has a strong connection to logical inference (Gardner et al., 2015), as the feature space considered by PRA consists of a restricted class of horn clauses found in the graph. While PRA can be applied to any link prediction task in a graph, its primary use has been in KB completion (Lao et al., 2012; Gardner et al., 2013; Gardner et al., 2014).

PRA is a two-step process, where the first step finds potential path types between node pairs to use as features in a statistical model, and the second step computes random walk probabilities associated with each path type and node pair (these are the values in a feature matrix). This second step is very computationally intensive, requiring time proportional to the average out-degree of the graph to the power of the path length for each cell in the computed feature matrix. In this paper we consider whether this computational effort is wellspent, or whether we might more profitably spend computation in other ways. We propose a new way of generating feature matrices over node pairs in a graph that aims to improve both the efficiency and the expressivity of the model relative to PRA.

Our technique, which we call subgraph feature extraction (SFE), is similar to only doing the first step of PRA. Given a set of node pairs in a graph, we first do a local search to characterize the graph around each node. We then run a set of feature extractors over these local subgraphs to obtain feature vectors for each node pair. In the simplest case, where the feature extractors only 
look for paths connecting the two nodes, the feature space is equivalent to PRA's, and this is the same as running PRA and binarizing the resultant feature vectors. However, because we do not have to compute random walk probabilities associated with each path type in the feature matrix, we can extract much more expressive features, including features which are not representable as paths in the graph at all. In addition, we can do a more exhaustive search to characterize the local graph, using a breadth-first search instead of random walks. SFE is a much simpler method than PRA for obtaining feature matrices over node pairs in a graph. Despite its simplicity, however, we show experimentally that it substantially outperforms PRA, both in terms of running time and prediction performance. SFE decreases running time over PRA by an order of magnitude, it improves mean average precision from .432 to .528 on the NELL KB, and it improves mean reciprocal rank from .850 to .933 .

In the remainder of this paper, we first describe PRA in more detail. We then situate our methods in the context of related work, and provide additional experimental motivation for the improvements described in this paper. We then formally define SFE and the feature extractors we used, and finally we present an experimental comparison between PRA and SFE on the NELL KB. The code and data used in this paper is available at http://rtw.ml.cmu.edu/emnlp2015_sfe/.

\section{The Path Ranking Algorithm}

The path ranking algorithm was introduced by Lao and Cohen (2010). It is a two-step process for generating a feature matrix over node pairs in a graph. The first step finds a set of potentially useful path types that connect the node pairs, which become the columns of the feature matrix. The second step then computes the values in the feature matrix by finding random walk probabilities as described below. Once the feature matrix has been computed, it can be used with whatever classification model is desired (or even incorporated as one of many factors in a structured prediction model), though almost all prior work with PRA simply uses logistic regression.

More formally, consider a graph $\mathcal{G}$ with nodes $\mathcal{N}$, edges $\mathcal{E}$, and edge labels $\mathcal{R}$, and a set of node pairs $\left(s_{j}, t_{j}\right) \in \mathcal{D}$ that are instances of some relationship of interest. PRA will generate a feature vector for each $\left(s_{j}, t_{j}\right)$ pair, where each feature is some sequence of edge labels $-e_{1}-e_{2}-\ldots-e_{l}$. If the edge sequence, or path type, corresponding to the feature exists between the source and target nodes in the graph, the value of that feature in the feature vector will be non-zero.

Because the feature space considered by PRA is so large, ${ }^{1}$ and because computing the feature values is so computationally intensive, the first step PRA must perform is feature selection, which is done using random walks over the graph. In this step of PRA, we find path types $\pi$ that are likely to be useful in predicting new instances of the relation represented by the input node pairs. These path types are found by performing random walks on the graph $\mathcal{G}$ starting at the source and target nodes in $\mathcal{D}$ and recording which paths connect some source node with its target. ${ }^{2}$ Note that these are two-sided, unconstrained random walks: the walks from sources and targets can be joined on intermediate nodes to get a larger set of paths that connect the source and target nodes. Once connectivity statistics have been computed in this way, $k$ path types are selected as features. Lao et al. (2011) use measures of the precision and recall of each feature in this selection, while Gardner et al. (2014) simply pick those most frequently seen.

Once a set of path features has been selected, the second step of PRA is to compute values for each cell in the feature matrix. Recall that rows in this matrix correspond to node pairs, and the columns correspond to the path types found in the first step. The cell value assigned by PRA is the probability of arriving at the target node of a node pair, given that a random walk began at the source node and was constrained to follow the path type: $\mathrm{p}(t \mid s, \pi)$. There are several ways of computing this probability. The most straightforward method is to use a path-constrained breadth-first search to exhaustively enumerate all possible targets given a source node and a path type, count how frequently each target is seen, and normalize the distribution. This calculates the desired probability exactly, but at the cost of doing a breadth-first search (with

\footnotetext{
${ }^{1}$ The feature space consists of the set of all possible edge label sequences, with cardinality $\sum_{i=1}^{l}|\mathcal{R}|^{i}$, assuming a bound $l$ on the maximum path length.

${ }^{2}$ A deterministic algorithm, such as a breadth-first search, could obviously be used here instead of random walks, and indeed Lao's original work did use a more exhaustive search. However, when moving to the larger graphs corresponding to the NELL and Freebase KBs, Lao (2011) (and all future work) switched to using random walks, because the graph was too large.
} 
complexity proportional to the average per-edgelabel out-degree to the power of the path length) per source node per path type.

There are three methods that can potentially reduce the computational complexity of this probability calculation. The first is to use random walks to approximate the probability via rejection sampling: for each path type and source node, a number of random walks are performed, attempting to follow the edge sequence corresponding to the path type. If a node is reached where it is no longer possible to follow the path type, the random walk is restarted. This does not reduce the time necessary to get an arbitrarily good approximation, but it does allow us to decrease computation time, even getting a fixed complexity, at the cost of accepting some error in our probability estimates. Second, Lao (2012) showed that when the target node of a query is known, the exponent can be cut in half by using a two-sided BFS. In this method, some careful bookkeeping is done with dynamic programming such that the probability can be computed correctly when the two-sided search meets at an intermediate node. Lao's dynamic programming technique is only applicable when the target node is known, however, and only cuts the exponent in half-this is still quite computationally intensive. Lastly, we could replace the BFS with a multiplication of adjacency matrices, which performs the same computation. The efficiency gain comes from the fact that we can just do the multiplication once per path type, instead of once per path type per source node. However, to correctly compute the probabilities for a (source, target) pair, we need to exclude from the graph the edge connecting that training instance. This means that the matrix computed for each path type should be different for each training instance, and so we either lose our efficiency gain or we accept incorrect probability estimates. In this work we use the rejection sampling technique.

As mentioned above, once the feature matrix has been computed in the second step of PRA, one can use any kind of classifier desired to learn a model and make predictions on test data.

\section{Related Work}

The task of knowledge base completion has seen a lot of attention in recent years, with entire workshops devoted to it (Suchanek et al., 2013). We will touch on three broad categories related to KB completion: the task of relation extraction, embedding methods for KB completion, and graph methods for KB completion.

Relation extraction. Relation extraction and knowledge base completion have the same goal: to predict new instances of relations in a formal knowledge base such as Freebase or NELL. The difference is that relation extraction focuses on determining what relationship is expressed by a particular sentence, while knowledge base completion tries to predict which relationships hold between which entities. A relation extraction system can be used for knowledge base completion, but typical KB completion methods do not make predictions on single sentences. This is easily seen in the line of work known as distantly-supervised relation extraction (Mintz et al., 2009; Hoffmann et al., 2011; Surdeanu et al., 2012); these models use the relation instances in a knowledge base as their only supervision, performing some heuristic mapping of the entities in text to the knowledge base, then using that mapping to train extractors for each relation in the $\mathrm{KB}$. The cost of using these methods is that is it generally difficult to incorporate richer features from the knowledge base when predicting whether a particular sentence expresses a relation, and so techniques that make fuller use of the KB can perform better on the KB completion task (Weston et al., 2013; Riedel et al., 2013).

Embedding methods for KB completion. There has been much recent work that attempts to perform KB completion by learning an embedded representation of entities and relations in the $\mathrm{KB}$ and then using these representations to infer missing relationships. Some of earliest work along these lines were the RESCAL model (Nickel et al., 2011) and Structured Embeddings (Bordes et al., 2011). These were soon followed by TransE (Bordes et al., 2013), Neural Tensor Networks (Socher et al., 2013), and many variants on all of these algorithms (Chang et al., 2014; García-Durán et al., 2014; Wang et al., 2014). These methods perform well when there is structural redundancy in the knowledge base tensor, but when the tensor (or individual relations in the tensor) has high rank, learning good embeddings can be challenging. The ARE model (Nickel et al., 2014) attempted to address this by only making the embeddings capture the residual of the tensor that cannot be readily predicted from the graph-based techniques mentioned below. 


\begin{tabular}{|l|l|r|}
\hline Dataset & Method & MAP \\
\hline \multirow{2}{*}{ Freebase } & Probabilities & .337 \\
\cline { 2 - 3 } & Binarized & .344 \\
\hline \hline \multirow{2}{*}{ NELL } & Probabilities & .303 \\
\cline { 2 - 3 } & Binarized & .319 \\
\hline
\end{tabular}

Table 1: Using binary feature values instead of random walk probabilities gives statistically indistinguishable performance. The $p$-value on the Freebase data is .55 , while it is .25 for NELL.

Graph-based methods for KB completion. A separate line of research into KB completion can be broadly construed as performing some kind of inference over graphs in order to predict missing instances in a knowledge base. Markov logic networks (Richardson and Domingos, 2006) fall into this category, as does ProPPR (Wang et al., 2013) and many other logic-based systems. PRA, the main subject of this paper, also fits in this line of work. Work specifically with PRA has ranged from incorporating a parsed corpus as additional evidence when doing random walk inference (Lao et al., 2012), to introducing better representations of the text corpus (Gardner et al., 2013; Gardner et al., 2014), and using PRA in a broader context as part of Google's Knowledge Vault (Dong et al., 2014). An interesting piece of work that combines embedding methods with graph-based methods is that of Neelakantan et al. (2015), which uses a recursive neural network to create embedded representations of PRA-style paths.

\section{Motivation}

We motivate our modifications to PRA with three observations. First, it appears that binarizing the feature matrix produced by PRA, removing most of the information gained in PRA's second step, has no significant impact on prediction performance in knowledge base completion tasks. We show this on the NELL KB and the Freebase KB in Table $1 .{ }^{3}$ The fact that random walk probabilities carry no additional information for this task over binary features is surprising, and it shows that the second step of PRA spends a lot of its computation for no discernible gain in performance.

Second, Neelakantan et al. (2015) presented ex-

${ }^{3}$ The NELL data and experimental protocol is described in Section 6.1. The Freebase data consists of 24 relations from the Freebase KB; we used the same data used by Gardner et al. (2014). periments showing a substantial increase in performance from using a much larger set of features in a PRA-like model. ${ }^{4}$ All of their experiments used binary features, so this is not a direct comparison of random walk probabilities versus binarized features, but it shows that increasing the feature size beyond the point that is computationally feasible with random walk probabilities seems useful. Additionally, they showed that using path bigram features, where each sequential pair of edges types in each path was added as an additional feature to the model, gave a significant increase in performance. These kind of features are not representable in the traditional formulation of PRA.

Lastly, the method used to compute the random walk probabilities-rejection sampling-makes the inclusion of more expressive features problematic. Consider the path bigrams mentioned above; one could conceivably compute a probability for a path type that only specifies that the last edge type in the path must be $r$, but it would be incredibly inefficient with rejection sampling, as most of the samples would end up rejected (leaving aside the additional issues of an unspecified path length). In contrast, if the features simply signify whether a particular path type exists in the graph, without any associated probability, these kinds of features are very easy to compute.

Given this motivation, our work attempts to improve both the efficiency and the expressivity of PRA by removing the second step of the algorithm. Efficiency is improved because the second step is the most computationally expensive, and expressivity is improved by allowing features that cannot be reasonably computed with rejection sampling. We show experimentally that the techniques we introduce do indeed improve performance quite substantially.

\section{Subgraph Feature Extraction}

In this section we discuss how SFE constructs feature matrices over node pairs in a graph using just a single search over the graph for each node (which is comparable to only using the first step of PRA). As outlined in Section 2, the first step of PRA does a series of random walks from each source and target node $\left(s_{j}, t_{j}\right)$ in a dataset $\mathcal{D}$. In

\footnotetext{
${ }^{4}$ Note that while Neelakantan et al. called the baseline they were comparing to "PRA", they only used the first step of the algorithm to produce path types, and thus did not really compare against PRA per se. It is their version of "PRA" that we formalize and expand as SFE in this work.
} 
PRA these random walks are used to find a relatively small set of potentially useful path types for which more specific random walk probabilities are then computed, at great expense. In our method, subgraph feature extraction (SFE), we stop after this first set of random walks and instead construct a binary feature matrix.

More formally, for each node $n$ in the data (where $n$ could be either a source node or a target node), SFE constructs a subgraph centered around that node using $k$ random walks. Each random walk that leaves $n$ follows some path type $\pi$ and ends at some intermediate node $i$. We keep all of these $(\pi, i)$ pairs as the characterization of the subgraph around $n$, and we will refer to this subgraph as $G_{n}$. To construct a feature vector for a source-target pair $\left(s_{j}, t_{j}\right)$, SFE takes the subgraphs $G_{s_{j}}$ and $G_{t_{j}}$ and merges them on the intermediate nodes $i$. That is, if an intermediate node $i$ is present in both $G_{s_{j}}$ and $G_{t_{j}}$, SFE takes the path types $\pi$ corresponding to $i$ and combines them (reversing the path type coming from the target node $t_{j}$ ). If some intermediate node for the source $s_{j}$ happens to be $t_{j}$, no combination of path types is necessary (and similarly if an intermediate node for the target $t_{j}$ is $s_{j}$-the path only needs to be reversed in this case). This creates a feature space that is exactly the same as that constructed by PRA: sequences of edge types that connect a source node to a target node. To construct the feature vector SFE just takes all of these combined path types as binary features for $\left(s_{j}, t_{j}\right)$. Note, however, that we need not restrict ourselves to only using the same feature space as PRA; Section 5.1 will examine extracting more expressive features from these subgraphs.

This method for generating a feature matrix over node pairs in a graph is much simpler and less computationally expensive than PRA, and from looking at Table 1 we would expect that it would perform on par with PRA with drastically reduced computation costs. Some experimentation shows that it is not that simple. Table 2 shows a comparison between PRA and SFE on 10 NELL relations. ${ }^{5}$ SFE has a higher mean average precision, but the difference is not statistically significant. There is a large variance in SFE's performance, and on some relations PRA performs better.

We examined the feature matrices computed

\footnotetext{
${ }^{5}$ The data and evaluation methods are described more fully in Section 6.1. These experiments were conducted on a different development split of the same data.
}

\begin{tabular}{|l|l|r|}
\hline Method & MAP & Ave. Features \\
\hline PRA & .3704 & 835 \\
\hline SFE & .4007 & 8275 \\
\hline
\end{tabular}

Table 2: Comparison of PRA and SFE on 10 NELL relations. The difference shown is not statistically significant.

by these methods and discovered that the reason for the inconsistency of SFE's improvement is because its random walks are all unconstrained. Consider the case of a node with a very high degree, say 1000. If we only do 200 random walks from this node, we cannot possibly get a complete characterization of the graph even one step away from the node. If a particularly informative path is $<$ CityInState, StateInCountry $>$, and both the city from which a random walk starts and the intermediate state node have very high degree, the probability of actually finding this path type using unconstrained random walks is quite low. This is the benefit gained by the path-constrained random walks performed by PRA; PRA leverages training instances with relatively low degree and aggregation across a large number of instances to find path types that are potentially useful. Once they are found, significant computational effort goes into discovering whether each path type exists for all $(s, t)$ pairs. It is this computational effort that allows the path type $<$ CityInState, StateInCOUNTRY $>$ to have a non-zero value even for very highly connected nodes.

How do we mitigate this issue, so that SFE can consistently find these path types? It seems the only option without resorting to a similar two-step process to what PRA uses is to do a more exhaustive search. PRA uses random walks to improve scalability on very large graphs, particularly because the second step of the algorithm is so expensive. However, if we are only doing a single search, and the graph fits in memory, a few steps of a breadth-first search (BFS) per node is not infeasible. We can make the BFS more tractable by excluding edge types whose fan out is too high. For example, at a type node in Freebase, there could be thousands of edges of type /TYPE/OBJECT/TYPE; if there are a large number of edges of the same type leaving a node, we do not include those edges in the BFS. Note that because the type node will still be counted as an intermediate node in the subgraph, we can still find paths that go through that 


\begin{tabular}{|l|r|r|r|}
\hline Method & MAP & Ave. Features & Time \\
\hline PRA & .3704 & 835 & $44 \mathrm{~min}$. \\
\hline SFE-RW & .4007 & 8275 & $6 \mathrm{~min}$. \\
\hline SFE-BFS & .4799 & 237853 & $5 \mathrm{~min}$. \\
\hline
\end{tabular}

Table 3: Comparison of PRA and SFE (with PRAstyle features) on 10 NELL relations. SFE-RW is not statistically better than PRA, but SFE-BFS is $(p<0.05)$.

node; we just do not continue searching if the outdegree of a particular edge type is too high.

When using a BFS instead of random walks to obtain the subgraphs $G_{s_{j}}$ and $G_{t_{j}}$ for each node pair, we saw a dramatic increase in the number of path type features found and a substantial increase in performance. ${ }^{6}$ These results are shown in Table 3; SFE-RW is our SFE implementation using random walks, and SFE-BFS uses a BFS.

\subsection{More expressive features}

The description above shows how to recreate the feature space used by PRA using our simpler subgraph feature extraction technique. As we have mentioned, however, we need not restrict ourselves to merely recreating PRA's feature space. Eliminating random walk probabilities allows us to extract a much richer set of features from the subgraphs around each node, and here we present the feature extractors we have experimented with. Figure 1 contains an example graph that we will refer to when describing these features.

PRA-style features. We explained these features in Section 5, but we repeat them here for consistency, and use the example to make the feature extraction process more clear. Relying on the notation introduced earlier, these features are generated by intersecting the subgraphs $G_{s}$ and $G_{t}$ on the intermediate nodes. That is, when the subgraphs share an intermediate node, we combine the path types found from the source and target to that node. In the example in Figure 1, there are two common intermediate nodes ("Barack Obama" and "Michelle Obama"), and combining the path types corresponding to those nodes gives the same path type: -ALIAS-"is married to"-ALIAS ${ }^{-1}$-.

Path bigram features. In Section 4, we mentioned that Neelakantan et al. (2015) experimented with using path bigrams as features. We

\footnotetext{
${ }^{6}$ One should not read too much into the decrease in running time between SFE-RW and SFE-BFS, however, as it was mostly an implementation detail.
}

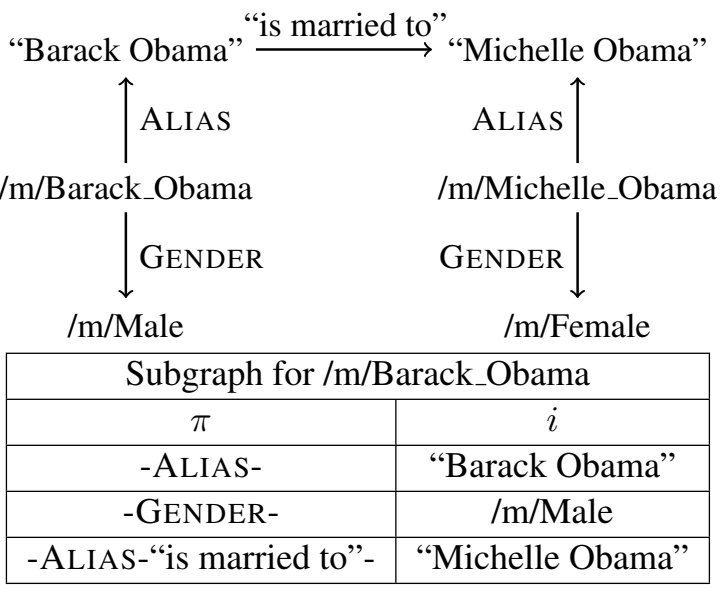

\begin{tabular}{|c|c|}
\hline \multicolumn{2}{|c|}{ Subgraph for /m/Michelle_Obama } \\
\hline$\pi$ & $i$ \\
\hline -ALIAS- & "Michelle Obama" \\
\hline -GENDER- & $/ \mathrm{m} /$ Female \\
\hline -ALIAS-"is married to"-1_- & "Barack Obama" \\
\hline
\end{tabular}

Figure 1: An example graph, with subgraphs extracted for two nodes.

include those features here as well. For any path $\pi$ between a source node $s$ and a target node $t$, we create a feature for each relation bigram in the path type. In the example in Figure 1, this would result in the features "BIGRAM:@START@-ALIAS", "BIGRAM:ALIAS-is married to", "BIGRAM:is married to-ALIAS", and "BIGRAM:ALIAS@END@”.

One-sided features. We use one-sided path to describe a sequence of edges that starts at a source or target node in the data, but does not necessarily terminate at a corresponding target or source node, as PRA features do. Following the notation introduced in Section 5, we use as features each $(\pi$, i) pair in the subgraph characterizations $G_{s}$ and $G_{t}$, along with whether the feature came from the source node or the target node. The motivation for these one-sided path types is to better model which sources and targets are good candidates for participating in a particular relation. For example, not all cities participate in the relation CITYCAPITALOFCOUNTRY, even though the domain of the relation is all cities. A city that has a large number of sports teams may be more likely to be a capital city, and these one-sided features could easily capture that kind of information.

Example one-sided features from the example in Figure 1 would be "SOURCE:GENDER-:male”, "TARGET:-GENDER-:female”, 
"SOURCE:-ALIAS-:Barack Obama", and "SOURCE:-ALIAS-is married to-:Michelle Obama".

One-sided feature comparisons. We can expand on the one-sided features introduced above by allowing for comparisons of these features in certain circumstances. For example, if both the source and target nodes have an age or gender encoded in the graph, we might profitably use comparisons of these values to make better predictions.

Drawing again on the notation from Section 5, we can formalize these features as analogous to the pairwise PRA features. To get the PRA features, we intersect the intermediate nodes $i$ from the subgraphs $G_{s}$ and $G_{t}$, and combine the path types $\pi$ when we find common intermediate nodes. To get these comparison features, we instead intersect the subgraphs on the path types, and combine the intermediate nodes when there are common path types. That is, if we see a common path type, such as -GENDER-, we will construct a feature representing a comparison between the intermediate node for the source and the target. If the values are the same, this information can be captured with a PRA feature, but it cannot be easily captured by PRA when the values are different.

In the example in Figure 1, there are two common path types: -ALIAS-, and -GENDER. The feature generated from the path type GENDER- would be "COMPARISON:-GENDER$: / \mathrm{m} /$ Male:/m/Female".

Vector space similarity features. Gardner et al. (2014) introduced a modification of PRA's random walks to incorporate vector space similarity between the relations in the graph. On the data they were using, a graph that combined a formal knowledge base with textual relations extracted from text, they found that this technique gave a substantial performance improvement. The vector space random walks only affected the second step of PRA, however, and we have removed that step in SFE. While it is not as conceptually clean as the vector space random walks, we can obtain a similar effect with a simple feature transformation using the vectors for each relation. We obtain vector representations of relations through factorization of the knowledge base tensor as did Gardner et al., and replace each edge type in a PRA-style path with edges that are similar to it in the vector space. We also introduce a special "any edge" symbol, and say that all other edge types are simi- lar to this edge type.

To reduce the combinatorial explosion of the feature space that this feature extractor creates, we only allow replacing one relation at a time with a similar relation. In the example graph in Figure 1, and assuming that "spouse of" is found to be similar to "is married to", some of the features extracted would be the following: "VECSIM:-ALIAS-is married toALIAS-", "VECSIM:-ALIAS-spouse of-AlIAS-", "VECSIM:-ALIAS-@ANY_REL@-ALIAS-", and "VECSIM:-@ANY_REL@-is married to-ALIAS". Note that the first of those features, "VECSIM:ALIAS-is married to-AliAS-", is necessary even though it just duplicates the original PRA-style feature. This allows path types with different but similar relations to generate the same features.

Any-Relation features. It turns out that much of the benefit gained from Gardner et al.'s vector space similarity features came from allowing any path type that used a surface edge to match any other surface edge with non-zero probability. ${ }^{7}$ To test whether the vector space similarity features give us any benefit over just replacing relations with dummy symbols, we add a feature extractor that is identical to the one above, assuming an empty vector similarity mapping. The features extracted from Figure 1 would thus be "ANYREL:-@ANY_REL@ is married to-ALIAS", "ANYREL:-ALIAS@ANY_REL@-ALIAS", “ANYREL:-ALIAS-is married to-@ANY_REL@”.

\section{Experiments}

Here we present experimental results evaluating the feature extractors we presented, and a comparison between SFE and PRA. As we showed in Section 5 that using a breadth-first search to obtain subgraphs is superior to using random walks, all of the experiments presented here use the BFS implementation of SFE.

\subsection{Data}

To evaluate SFE and the feature extractors we introduced, we learned models for 10 relations in the NELL KB. We used the same data as Gardner et al. (2014), using both the formal KB relations and the surface relations extracted from text in our

\footnotetext{
${ }^{7}$ Replacing all surface edges with a single dummy relation gives performance close to vector space PRA. The vector space walks do statistically outperform this, but the extra gain is small.
} 
graph. We used logistic regression with elastic net (L1 and L2) regularization. We tuned the L1 and L2 parameters for each method on a random development split of the data, then used a new split of the data to run the final tests presented here.

The evaluation metrics we use are mean average precision (MAP) and mean reciprocal rank (MRR). We judge statistical significance using a paired permutation test, where the average preci$\operatorname{sion}^{8}$ on each relation is used as paired data.

\subsection{On Obtaining Negative Evidence}

One important practical issue for most uses of PRA is the selection of negative examples for training a model. Typically a knowledge base only contains positive examples of a relation, and it is not clear a priori what the best method is for obtaining negative evidence. Prior work with PRA makes a closed world assumption, treating any $(s$, $t$ ) pair not seen in the knowledge base as a negative example. Negative instances are selected when performing the second step of PRA-if a random walk from a source ends at a target that is not a known correct target for that source, that sourcetarget pair is used as a negative example.

SFE only scores (source, target) pairs; it has no mechanism similar to PRA's that will find potential targets given a source node. We thus need a new way of finding negative examples, both at training time and at test time. We used a simple technique to find negative examples from a graph given a set of positive examples, and we used this to obtain the training and testing data used in the experiments below. Our technique takes each source and target node in the given positive examples and finds other nodes in the same category that are close in terms of personalized page rank (PPR). We then sample new (source, target) pairs from these lists of similar nodes, weighted by their PPR score (while also allowing the original source and target to be sampled). These become our negative examples, both at training and at testing time.

Because this is changing the negative evidence available to PRA at training time, we wanted to be sure we were not unfairly hindering PRA in our comparisons. If it is in fact better to let PRA find its own negative examples at training time, instead of the ones sampled based on personalized page rank, then we should let PRA get its own nega-

\footnotetext{
${ }^{8}$ Average precision is equivalent to the area under a precision/recall curve.
}

\begin{tabular}{|l|r|}
\hline Method & MAP \\
\hline PRA's random walks & .359 \\
\hline PPR-based sampling & .363 \\
\hline
\end{tabular}

Table 4: Comparing methods for obtaining negative evidence available at training time. The difference seen is not statistically significant $(p=.77)$.

tive evidence. We thus ran an experiment to see under which training regime PRA performs better. We created a test set with both positive and negative examples as described in the paragraph above, and at training time we compared two techniques: (1) letting PRA find its own negative examples through its random walks, and (2) only using the negative examples selected by PPR. As can be seen in Table 4, the difference between the two training conditions is very small, and it is not statistically significant. Because there is no significant difference between the two conditions, in the experiments that follow we give both PRA and SFE the same training data, created through the PPR-based sampling technique described above.

\subsection{Results}

We first examine the effect of each of the feature types introduced in Section 5.1. The results are shown in Table 5. We can see that, for this data, the comparisons and one-sided features did not improve performance (and the decreases are not statistically significant). Bigram features do appear to improve performance, though the improvement was not consistent enough across relations to achieve statistical significance. The vector similarity features do improve performance, with $p$-values hovering right at 0.05 when comparing against only PRA features and PRA + bigram features. The any rel features, however, do statistically improve over all other methods $(p<=0.01)$ except the PRA + vec sim result $(p=.21)$.

Finally, we present a comparison between PRA, PRA with vector space random walks, and the best SFE result from the ablation study. This is shown in Table 6. SFE significantly outperforms PRA, both with and without the vector space random walks presented by Gardner et al. (2014).

\subsection{Discussion}

When using only PRA-style features with SFE, the highest weighted features were almost always those of the form -ALIAS-[some textual relation]ALIAS $^{-1}$-. For example, for the relation WRITER- 


\begin{tabular}{|l|r|r|r|}
\hline Feature Types & MAP & MRR & Features \\
\hline PRA-style features & .431 & .806 & $240 \mathrm{k}$ \\
\hline + Comparisons & .405 & .833 & $558 \mathrm{k}$ \\
\hline + One-sided & .389 & .800 & $1,227 \mathrm{k}$ \\
\hline + One-sided + Comps. & .387 & .817 & $1,544 \mathrm{k}$ \\
\hline + Bigrams & .483 & 1.00 & $320 \mathrm{k}$ \\
\hline + Vector similarity & .514 & .910 & $3,993 \mathrm{k}$ \\
\hline + Bigrams + vec sim. & .490 & .950 & $4,073 \mathrm{k}$ \\
\hline + Any Rel & $\mathbf{. 5 2 8}$ & .933 & $649 \mathrm{k}$ \\
\hline
\end{tabular}

Table 5: SFE feature ablation study. All rows use PRA features. PRA + any rel is statistically better than all other methods except PRA + vec sim, and most of the other differences are not significant.

\begin{tabular}{|l|r|r|}
\hline Method & MAP & MRR \\
\hline PRA & .362 & .717 \\
\hline Vector space PRA & .432 & .850 \\
\hline SFE (PRA + any rel features) & $\mathbf{. 5 2 8}$ & .933 \\
\hline
\end{tabular}

Table 6: Results of final comparison between SFE and PRA, with and without vector space similarity features. SFE is statistically better than both PRA methods $(p<0.005)$.

WROTEBOOK, the textual relations used in this feature might be "wrote", "describes in", "writes in", and "expresses in". These are the same feature types that PRA itself finds to have the highest weight, also, though SFE finds many more of them than PRA does, as PRA has to do aggressive feature selection. For this particular dataset, where the graph consists of edges from a formal $\mathrm{KB}$ mixed with edges from extracted textual relations, these kinds of features are by far the most useful, and most of the improvements seen by the additional feature types we used with SFE come from more compactly encoding these features.

For example, the path bigram features can encode the fact that there exists a path from the source to the target that begins or ends with an ALIAS edge. This captures in just two features all path types of the form -ALIAS-[some textual relation]-ALIAS ${ }^{-1}-$, and those two bigram features are almost always the highest weighted features in models where they are used.

However, the bigram features do not capture those path types exactly. The Any-Rel features were designed in part specifically for this path type, and they capture it exactly with a single feature. For all 10 relations, the feature "ANYREL:ALIAS-@ANY_REL@-ALIAS ${ }^{-1}$ " is the highest weighted feature. This is because, for the relations we experimented with, knowing that some relationship is expressed in text between a particular pair of $\mathrm{KB}$ entities is a very strong indication of a single KB relation. There are only so many possible relationships between cities and countries, for instance. These features are much less informative between entity types where more than one relation is possible, such as between people.

While the bigram and any-rel features capture succintly whether textual relations are present between two entities, the one-sided features are more useful for determining whether an entity fits into the domain or range of a particular relation. We saw a few features that did this, capturing finegrained entity types. Most of the features, however, tended towards memorizing (and thus overfitting) the training data, as these features contained the names of the training entities. We believe this overfitting to be the main reason these features did not improve performance, along with the fact that the relations we tested do not need much domain or range modeling (as opposed to, e.g., SpouseOf or CityCapital OfCountry).

\section{Conclusion}

We have explored several practical issues that arise when using the path ranking algorithm for knowledge base completion. An analysis of several of these issues led us to propose a simpler algorithm, which we called subgraph feature extraction, which characterizes the subgraph around node pairs and extracts features from that subgraph. SFE is both significantly faster and performs better than PRA on this task. We showed experimentally that we can reduce running time by an order of magnitude, while at the same time improving mean average precision from .432 to .528 and mean reciprocal rank from .850 to .933 . This thus constitutes the best published results for knowledge base completion on NELL data. The code and data used in the experiments in this paper are available at http://rtw.ml.cmu.edu/emnlp2015_sfe/.

\section{Acknowledgements}

This work was supported in part by NSF grant IIS1247489, in part by support as a Yahoo! Fellow, and in part by DARPA contract FA87501320005. 


\section{References}

Jonathan Berant, Andrew Chou, Roy Frostig, and Percy Liang. 2013. Semantic parsing on freebase from question-answer pairs. In $E M N L P$, pages 1533 1544.

Kurt Bollacker, Colin Evans, Praveen Paritosh, Tim Sturge, and Jamie Taylor. 2008. Freebase: a collaboratively created graph database for structuring human knowledge. In Proceedings of SIGMOD.

Antoine Bordes, Jason Weston, Ronan Collobert, Yoshua Bengio, et al. 2011. Learning structured embeddings of knowledge bases. In $A A A I$.

Antoine Bordes, Nicolas Usunier, Alberto GarciaDuran, Jason Weston, and Oksana Yakhnenko. 2013. Translating embeddings for modeling multirelational data. In Advances in Neural Information Processing Systems, pages 2787-2795.

Kai-Wei Chang, Wen-tau Yih, Bishan Yang, and Christopher Meek. 2014. Typed tensor decomposition of knowledge bases for relation extraction. In Proceedings of the 2014 Conference on Empirical Methods in Natural Language Processing (EMNLP), pages 1568-1579.

Eunsol Choi, Tom Kwiatkowski, and Luke Zettlemoyer. 2015. Scalable semantic parsing with partial ontologies. In Proceedings of ACL.

Xin Dong, Evgeniy Gabrilovich, Geremy Heitz, Wilko Horn, Ni Lao, Kevin Murphy, Thomas Strohmann, Shaohua Sun, and Wei Zhang. 2014. Knowledge vault: A web-scale approach to probabilistic knowledge fusion. In Proceedings of the 20th ACM SIGKDD international conference on Knowledge discovery and data mining, pages 601-610. ACM.

Alberto García-Durán, Antoine Bordes, and Nicolas Usunier. 2014. Effective blending of two and threeway interactions for modeling multi-relational data. In Machine Learning and Knowledge Discovery in Databases, pages 434-449. Springer.

Matt Gardner, Partha Talukdar, Bryan Kisiel, and Tom Mitchell. 2013. Improving learning and inference in a large knowledge-base using latent syntactic cues. In Proceedings of EMNLP. Association for Computational Linguistics.

Matt Gardner, Partha Talukdar, Jayant Krishnamurthy, and Tom Mitchell. 2014. Incorporating vector space similarity in random walk inference over knowledge bases. In Proceedings of EMNLP. Association for Computational Linguistics.

Matt Gardner, Partha Talukdar, and Tom Mitchell. 2015. Combining vector space embeddings with symbolic logical inference over open-domain text. In 2015 AAAI Spring Symposium Series.
Raphael Hoffmann, Congle Zhang, Xiao Ling, Luke Zettlemoyer, and Daniel S Weld. 2011. Knowledgebased weak supervision for information extraction of overlapping relations. In Proceedings of the 49th Annual Meeting of the Association for Computational Linguistics: Human Language TechnologiesVolume 1, pages 541-550. Association for Computational Linguistics.

Jayant Krishnamurthy and Tom M Mitchell. 2012. Weakly supervised training of semantic parsers. In Proceedings of the 2012 Joint Conference on Empirical Methods in Natural Language Processing and Computational Natural Language Learning, pages 754-765. Association for Computational Linguistics.

Ni Lao and William W Cohen. 2010. Relational retrieval using a combination of path-constrained random walks. Machine learning, 81(1):53-67.

Ni Lao, Tom Mitchell, and William W Cohen. 2011. Random walk inference and learning in a large scale knowledge base. In Proceedings of EMNLP. Association for Computational Linguistics.

Ni Lao, Amarnag Subramanya, Fernando Pereira, and William W Cohen. 2012. Reading the web with learned syntactic-semantic inference rules. In Proceedings of EMNLP-CoNLL.

Ni Lao. 2012. Efficient Random Walk Inference with Knowledge Bases. Ph.D. thesis, Carnegie Mellon University.

Pablo N. Mendes, Max Jakob, and Christian Bizer. 2012. Dbpedia for nlp: A multilingual cross-domain knowledge base. In Proceedings of the Eighth International Conference on Language Resources and Evaluation (LREC'12).

Mike Mintz, Steven Bills, Rion Snow, and Dan Jurafsky. 2009. Distant supervision for relation extraction without labeled data. In Proceedings of the Joint Conference of the 47th Annual Meeting of the $A C L$ and the 4th International Joint Conference on Natural Language Processing of the AFNLP, pages 1003-1011. Association for Computational Linguistics.

Tom M. Mitchell, William Cohen, Estevam Hruschka, Partha Talukdar, Justin Betteridge, Andrew Carlson, Bhavana Dalvi, Matthew Gardner, Bryan Kisiel, Jayant Krishnamurthy, Ni Lao, Kathryn Mazaitis, Thahir Mohamed, Ndapa Nakashole, Emmanouil Antonios Platanios, Alan Ritter, Mehdi Samadi, Burr Settles, Richard Wang, Derry Wijaya, Abhinav Gupta, Xinlei Chen, Abulhair Saparov, Malcolm Greaves, and Joel Welling. 2015. Neverending learning. In $A A A I$ 2015. Association for the Advancement of Artificial Intelligence.

Arvind Neelakantan, Benjamin Roth, and Andrew McCallum. 2015. Compositional vector space models for knowledge base completion. In ACL 2015. Association for Computational Linguistics. 
Maximilian Nickel, Volker Tresp, and Hans-Peter Kriegel. 2011. A three-way model for collective learning on multi-relational data. In Proceedings of the 28th international conference on machine learning (ICML-11), pages 809-816.

Maximilian Nickel, Xueyan Jiang, and Volker Tresp. 2014. Reducing the rank in relational factorization models by including observable patterns. In $A d$ vances in Neural Information Processing Systems, pages $1179-1187$.

Matthew Richardson and Pedro Domingos. 2006. Markov logic networks. Machine learning, 62(12):107-136.

Sebastian Riedel, Limin Yao, Andrew McCallum, and Benjamin M Marlin. 2013. Relation extraction with matrix factorization and universal schemas. In Proceedings of NAACL-HLT.

Richard Socher, Danqi Chen, Christopher D Manning, and Andrew Ng. 2013. Reasoning with neural tensor networks for knowledge base completion. In $\mathrm{Ad}$ vances in Neural Information Processing Systems, pages 926-934.

Fabian Suchanek, James Fan, Raphael Hoffmann, Sebastian Riedel, and Partha Pratim Talukdar. 2013. Advances in automated knowledge base construction. SIGMOD Records journal, March.

Mihai Surdeanu, Julie Tibshirani, Ramesh Nallapati, and Christopher D Manning. 2012. Multi-instance multi-label learning for relation extraction. In Proceedings of the 2012 Joint Conference on Empirical Methods in Natural Language Processing and Computational Natural Language Learning, pages 455465. Association for Computational Linguistics.

William Yang Wang, Kathryn Mazaitis, and William W. Cohen. 2013. Programming with personalized pagerank: A locally groundable first-order probabilistic logic. In Proceedings of the 22Nd ACM International Conference on Conference on Information \&\#38; Knowledge Management, CIKM '13, pages 2129-2138, New York, NY, USA. ACM.

Zhen Wang, Jianwen Zhang, Jianlin Feng, and Zheng Chen. 2014. Knowledge graph embedding by translating on hyperplanes. In Proceedings of the TwentyEighth AAAI Conference on Artificial Intelligence, pages 1112-1119.

Robert West, Evgeniy Gabrilovich, Kevin Murphy, Shaohua Sun, Rahul Gupta, and Dekang Lin. 2014. Knowledge base completion via search-based question answering. In $W W W$.

Jason Weston, Antoine Bordes, Oksana Yakhnenko, and Nicolas Usunier. 2013. Connecting language and knowledge bases with embedding models for relation extraction. In Proceedings of EMNLP. 